\title{
Physiotherapy, aerobics, and training devices reduced pain intensity and frequency in chronic low back pain
}

\author{
Mannion AF, Müntener M, Taimela S, et al. Comparison of three active therapies for chronic low back pain: results of a \\ randomized clinical trial with one-year follow-up. Rheumatology $2001 \mathrm{Jul} ; 40: 772-8$.

\section{QUESTION: In patients with chronic low back pain (LBP), are physiotherapy, specific conditioning with training devices, and aerobics equally effective for reducing pain and disability?}

Swiss National Science

Foundation and

Schulthess Klinik

Research Fund.

Design

Randomised \{allocation concealed $*\} \uparrow$, \{unblinded $\} \uparrow *$ controlled trial with 12 months of follow up.

A F Mannion

Schulthess Klinik,

Zurich, Switzerland.

afm@kws.ch.

\section{Setting}

A hospital in Switzerland.

Physiotherapy $v$ aerobics $v$ devices in chronic low back pain at 12 months

\begin{tabular}{|c|c|c|c|c|}
\hline \multirow[b]{2}{*}{ Outcomes } & \multicolumn{3}{|c|}{ Mean score (baseline) } & \multirow[b]{2}{*}{ p Value§ } \\
\hline & Physiotherapy§ & Aerobics & Devices & \\
\hline Highest pain (range 0 to 10 ) & $4.8(6.5)$ & $4.7(6.4)$ & $4.5(6.6)$ & 0.99 \\
\hline Average pain (range 0 to 10 ) & $3.2(4.4)$ & $3.2(4.1)$ & $2.9(4.2)$ & 0.90 \\
\hline Pain frequency (range 1 to 4 ) & $3.0(3.4)$ & $2.9(3.4)$ & $2.8(3.4)$ & 0.82 \\
\hline Disability (range 0 to 24 ) & $7.4(8.0)$ & $6.2(7.6)$ & $5.8(8.3)$ & $0.03^{* \star}$ \\
\hline
\end{tabular}

§Used a repeated measures analysis of variance.

ๆHigher scores $=$ greater disability.

${ }^{* *}$ The physiotherapy group differed from the aerobics and devices groups in pattern of change over the course of the study.

\section{COMMENTARY}

Chronic LBP is a serious condition that is associated with substantial economic and societal costs. The well designed study by Mannion et al compared the relative effectiveness of 3 therapeutic programmes for LBP. The investigators did not include an untreated control group, but previous systematic reviews have suggested that active physical measures are superior to usual care by practitioners. ${ }^{1}$ Given the lengthy duration of LBP in patients in the study by Mannion et al, observed improvements would probably not be expected merely as part of the natural history of the disorder. Moreover, because participants were volunteers who were motivated to reply to an advertisement, the degree of observed improvement may be greater than might be attained in typical clinical practice.

Although most outcome measures were equally effective among the groups immediately after each 3 month programme, self reported disability and psychological disturbance levels in the physiotherapy group were shown to increase at 6 months of follow up. This finding deserves further study to determine whether a clinically important difference exists between the outcomes that might be expected from these modalities. Overall, this study confirms the work of other researchers, suggesting that different physical methods of chronic LBP treatment are similarly effective. ${ }^{2}$

Ultimately, it seems that some form of active treatment is superior to self care in chronic LBP. Clinicians should tailor their treatment approaches according to availability, cost, and patient preference.

Eric W Vogel, MD MCP Hahnemann University School of Medicine Philadelphia, Pennsylvania, USA

1 van Tulder MW, Malmivaara A, Esmail R, et al. Exercise therapy for low back pain. Cochrane Database Syst Rev 2001;(4):CD000335 (latest version 9 Feb 2000).

2 Torstensen TA, Ljunggren AE, Meen HD, et al. Efficiency and costs of medical exercise therapy, conventional physiotherapy, and self-exercise in patients with chronic low back pain. A pragmatic, randomized, single-blinded, controlled trial with 1-year follow-up. Spine 1998;23:2616-24.

\section{Patients}

148 patients (mean age 45 y, $57 \%$ women, mean LBP duration $11 \mathrm{y}$ ) who were $<65$ years of age and had $>3$ months of LBP with or without referred pain serious enough to require medical attention or absence from work. Exclusion criteria included constant or persistent severe pain, pregnancy, previous spinal surgery, and current nerve-root entrapment accompanied by neurological deficit. $86 \%$ of patients completed follow up.

\section{Intervention}

Patients were allocated to 1 of 3 groups: physiotherapy $(\mathrm{n}=49)$ in 30 minute individual sessions with instruction on ergonomic principles and home exercises; lowimpact aerobics $(\mathrm{n}=50)$ in 1 hour sessions; or training devices $(n=49)$ in 1 hour sessions with specific trunk muscle reconditioning on training machines. All treatments were given for 3 months.

\section{Main outcome measures}

Pain intensity (visual analogue scale, score range 0 to 10 ), frequency of pain (pain free $=1$, sporadic $=2$, often $=3$, or continuous $=4$ ), and disability (Roland and Morrisł, score range 0 to 24 ).

\section{Main results}

Analysis was by intention to treat. Decreases in highest and average pain intensity scores and pain frequency were seen in each group, but the groups did not differ in the extent of their decreases (12 mo data are in the table). The devices and aerobics groups showed reductions in disability scores over time, but the physiotherapy group (which had an increase from post-treatment values in disability scores at $6 \mathrm{mo}$ ) differed statistically from the other groups (12 mo data are in the table).

\section{Conclusions}

In patients with chronic low back pain, physiotherapy, specific conditioning with training devices, and aerobics were similarly effective for reducing the pain intensity score and pain frequency. In contrast to the physiotherapy group, the aerobics and devices groups maintained their post-treatment reductions in disability after 12 months of follow up.

*See glossary.

†Information provided by author.

\$Roland M, Morris R. Spine 1983;8:141-4. 